\title{
Imaging Microglial Activation with TSPO PET: Lighting Up Neurologic Diseases?
}

\author{
Lucy Vivash and Terence J. O’Brien
}

Department of Medicine, Royal Melbourne Hospital, University of Melbourne, Royal Parade, Parkville, Victoria, Australia

\begin{abstract}
Neuroinflammation is implicated in the pathogenesis of a wide range of neurologic and neuropsychiatric diseases. For over 20 years, ${ }^{11} \mathrm{C}-\mathrm{PK} 11195 \mathrm{PET}$, which aims to image expression of the translocator protein (TSPO) on activated microglia in the brain, has been used in preclinical and clinical research to investigate neuroinflammation in vivo in patients with brain diseases. However, ${ }^{11} \mathrm{C}-\mathrm{PK} 11195$ suffers from two major limitations: its low brain permeability and high nonspecific and plasma binding results in a low signal-to-noise ratio, and the use of ${ }^{11} \mathrm{C}$ restricts its use to PET research centers and hospitals with an on-site cyclotron. In recent years, there has been a great deal of work into the development of new TSPO-specific PET radiotracers. This work has focused on fluorinated radiotracers, which would enable wider use and improved signal-to-noise ratios. These radiotracers have been utilized in preclinical and clinical studies of several neurologic diseases with varying degrees of success. Unfortunately, the application of these second-generation TSPO radiotracers has revealed additional problems, including a polymorphism that affects TSPO binding. In this review, the developments in TSPO imaging are discussed, and current limitations and suggestions for future directions are explored.
\end{abstract}

Key Words: positron emission tomography; TSPO; microglia; neurology; ${ }^{11} \mathrm{C}-\mathrm{PK} 11195$

J Nucl Med 2016; 57:165-168

DOI: 10.2967/jnumed.114.141713

The translocator protein (TSPO), originally designated the peripheral benzodiazepine receptor, is an 18-kDa protein found on the outer mitochondrial membrane. TSPO is expressed in a variety of tissues throughout the body but is prominently upregulated with inflammation. In the healthy brain there is low TSPO expression on microglia, the primary immune cells in the central nervous system. However, when microglia are activated in response to an injury, TSPO expression is markedly upregulated. As a result, the expression of TSPO has been considered a marker of neuroinflammation, and its increased brain expression is associated with

Received Apr. 22, 2015; revision accepted Dec. 22, 2015.

For correspondence or reprints contact: Lucy Vivash, Melbourne Brain Centre, 4 Centre, Royal Melbourne Hospital, Grattan St., Parkville, VIC, 3050, Australia.

E-mail: lucy.vivash@mh.org.au

Published online Dec. 23, 2015.

COPYRIGHT (c) 2016 by the Society of Nuclear Medicine and Molecular Imaging, Inc. numerous neurologic diseases (1). For this reason, imaging expression of TSPO with PET has been developed as a potentially clinically applicable tool for in vivo imaging of the patterns of neuroinflammation. TSPO PET imaging holds promise both for increasing understanding of the role of neuroinflammation in brain diseases and as a tool for assessing the efficacy of novel therapies. The structure and pharmacology of TSPO have been reviewed in detail by Scarf and Kassiou (2).

\section{${ }^{11}$ C-PK11195}

The most widely used TSPO PET tracer has been ${ }^{11} \mathrm{C}$ PK11195, an isoquinoline carboxamide that is a selective antagonist for TSPO. ${ }^{11} \mathrm{C}-\mathrm{PK} 11195$ was initially used as a racemate, but later studies found that the $R$-enantiomer binds with a 2-fold greater affinity than the $S$-enantiomer. Therefore, subsequent studies have utilized ${ }^{11} \mathrm{C}-(R)$-PK11195 PET to investigate neuroinflammation in several neurologic and neurooncologic diseases (3).

However, several major limitations have restricted the widespread use of ${ }^{11} \mathrm{C}-\mathrm{PK} 11195$ in the imaging of neuroinflammation in research and clinical practice. First, ${ }^{11} \mathrm{C}-\mathrm{PK} 11195$ has relatively low brain permeability and high nonspecific and plasma protein binding, which result in a low signal-to-noise ratio and low specificity in PET images. This means that ${ }^{11} \mathrm{C}-\mathrm{PK} 11195$ PET is not able to detect subtle alterations in neuroinflammation or to accurately track temporal changes in neuroinflammation. Second, the use of the short-half-life ${ }^{11} \mathrm{C}$ as the radioisotope restricts use of the radiotracer to PET centers with an on-site cyclotron. Thus, recent studies have focused on the development of fluorinated $\left({ }^{18} \mathrm{~F}\right)$ TSPO radiotracers, which have the advantage of a longer half-life and therefore do not require an on-site cyclotron, improving accessibility of the radioisotope to researchers and clinicians, considerably reducing costs, and improving signal-to-noise ratios.

\section{SECOND- AND THIRD-GENERATION TSPO RADIOTRACERS IN NEUROLOGIC DISEASES}

After the synthesis of multiple different fluorinated TSPO-specific radiotracers (Table 1 (4)), numerous preclinical and early clinical studies were conducted on a range of neurologic and oncologic diseases. Preclinical studies on rat glioma models have shown high uptake of ${ }^{18} \mathrm{~F}$-PBR06 and ${ }^{18} \mathrm{~F}-\mathrm{DPA} 714$ in tumor compared with the contralateral 
TABLE 1

A Summary of the Newer Generation of TSPO Radiotracers

\begin{tabular}{|c|c|c|}
\hline Chemical class & Radioligand & Binding affinity \\
\hline Indole acetamides & ${ }^{11} \mathrm{C}-S S R 180575$ & - \\
\hline Vinca alkaloids & ${ }^{11} \mathrm{C}$-vinpocetine & - \\
\hline \multirow[t]{5}{*}{ Phenoxyarylacetamides } & ${ }^{11} \mathrm{C}-\mathrm{PBR} 28$ & $2.2-52 \mathrm{nM}$ in humans \\
\hline & ${ }^{18}$ F-FEDAA1106 & - \\
\hline & ${ }^{18} \mathrm{~F}-\mathrm{PBR} 06$ & - \\
\hline & ${ }^{18}$ F-FEPPA & - \\
\hline & ${ }^{11} \mathrm{C}-\mathrm{DAA} 1106$ & $<1 \mathrm{nM}$ in humans \\
\hline \multirow[t]{2}{*}{ Imidazopyridine acetamides } & ${ }^{11} \mathrm{C}-\mathrm{DPA} 713$ & $1.3 \mathrm{nM}$ in mice \\
\hline & ${ }^{18} \mathrm{~F}-\mathrm{PBR} 111$ & - \\
\hline \multirow[t]{2}{*}{ Dihydro-9H-purinacetamides } & ${ }^{11} \mathrm{C}-\mathrm{DAC}$ & - \\
\hline & ${ }^{11} \mathrm{C}-\mathrm{AC}-5216$ & - \\
\hline Pyrazolopyrimidines & ${ }^{18} \mathrm{~F}-\mathrm{DPA}-714$ & - \\
\hline
\end{tabular}

hemisphere $(5,6)$. Both tracers have shown a high degree of specific binding to TSPO (75\% displaceable binding for ${ }^{18}$ F-PBR06, 60\% for ${ }^{18}$ F-DPA714). However, TSPO imaging is unable to differentiate expression on tumor cells from expression on inflammatory cells surrounding the tumor-a major limitation for the clinical application of TSPO imaging of brain tumors.

TSPO expression has also been investigated using PET in preclinical and clinical studies of Alzheimer disease. Microglial activation has been observed in mouse models of Alzheimer disease and in patients $(7,8)$. However, it has yet to be firmly established whether the microglial activation observed corresponds with amyloid or tau distribution or represents an independent pathologic distribution. Further, other clinical studies have failed to differentiate Alzheimer disease patients from age-matched controls $(9,10)$. This disparity indicates the importance of trialing a variety of radiotracers for appropriate sensitivity and specificity.

TSPO PET imaging has also been used by stroke researchers to investigate the role and time course of neuroinflammation after an acute cerebral infarction. Numerous TSPO-specific radiotracers have been used to image the core and periinfarct region in rat models of ischemia, demonstrating specific uptake in the region of an ischemic infarct, in agreement with ex vivo autoradiography studies (11). In patients, increased uptake of the tracer ${ }^{11} \mathrm{C}$-vinpocetine has been observed in both the infarct region and the periinfarct region compared with the contralateral hemisphere (12). Uptake of ${ }^{11} \mathrm{C}$-vinpocetine was higher than that of ${ }^{11}$ C-PK11195 and, unlike ${ }^{11}$ C-PK11195, was also able to differentiate the infarct from the periinfarct region (with greater uptake observed in the periinfarct region than in the infarct). However, the authors commented on the low affinity of ${ }^{11} \mathrm{C}$ vinpocetine for TSPO, which may limit its future clinical use.

Possibly the greatest clinical opportunity for in vivo imaging of neuroinflammation with TSPO PET is in patients with multiple sclerosis. Both acute inflammation and chronic neuroinflammation are implicated in the pathogenesis and clinical manifestation of this disease; however, this correlation has been difficult to study directly because of a lack of methods to assess neuroinflammation in vivo in humans. Therapies for patients with multiple sclerosis are directed against aspects of the inflammatory process, and therefore an accurate, clinically applicable tool to measure neuroinflammation could provide a valuable biomarker to assess the efficacy of treatment against disease progression. Such a biomarker would have great value both in drug development and in managing the treatment of individual patients. To date, a small number of preclinical and clinical studies have been reported. In the experimental autoimmune encephalomyelitis animal model, spinal TSPO expression has been imaged using numerous different PET radiotracers. When the radiotracers ${ }^{11} \mathrm{C}$-DAC and ${ }^{18} \mathrm{~F}-\mathrm{GE} 180$ were used with PET, increased TSPO expression was observed in the brains of these mice, compared with controls, and this expression was shown to correlate with expression on ex vivo autoradiography and immunohistochemistry studies (13). Importantly, after treatment with immunosuppressant drugs, radiotracer uptake was greatly reduced, indicating the future use of TSPO PET as a noninvasive biomarker for evaluating and monitoring the effect of multiple sclerosis therapies on disease activity $(13,14)$. Clinical studies on patients with multiple sclerosis have shown increased radiotracer uptake in lesional and perilesional white matter compared with nonlesional white matter using ${ }^{18} \mathrm{~F}$ PBR111 and ${ }^{11} \mathrm{C}-\mathrm{PBR} 28$ but not ${ }^{18} \mathrm{~F}-\mathrm{FEDAA} 1106$ (15-17), with radiotracer uptake shown to correlate positively with disease duration or severity, indicating potential utility for TSPO PET in monitoring disease activity.

\section{LIMITATIONS OF NEW TSPO RADIOTRACERS}

Despite studies showing promise for these newer TSPO radiotracers, significant issues have emerged that may limit the application of these tracers for routine clinical use. Early work using ${ }^{11} \mathrm{C}$-PBR28 reported a proportion of patients who were "nonbinders," meaning the radiotracer 
did not bind to TSPO. Further studies found that this nonbinder-group problem was not limited to ${ }^{11} \mathrm{C}-\mathrm{PBR} 28$ but affected all studied second-generation TSPO radiotracers except ${ }^{11} \mathrm{C}$-PK11195 (18). Three distinct binder states have been identified: high-, mixed-, and low-affinity binders (18), which have been found to be determined by a single polymorphism (rs6971) in exon 4 of the TSPO gene causing an alanine-to-threonine substitution (19). The presence of the predominant form, Ala/Ala, is associated with highaffinity binding, whereas Ala/Thr is associated with mixedaffinity binding and Thr/Thr with low-affinity binding. Initially identified using nonradiolabeled PBR28 (19), this polymorphism has also been confirmed for ${ }^{18} \mathrm{~F}-\mathrm{PBR} 111$ (Fig. 1 (20)). Importantly, this polymorphism (and consequently binder status) can be identified by genetic analysis of leukocytes, allowing stratification of subjects, and can subsequently account for binder status in quantitation of TSPO PET studies using second-generation radiotracers. However, in those patients who are low-affinity binders, TSPO PET images are of significantly lower quality, and the clinical usefulness of this approach is therefore limited in these patients.

A second concern for the value of TSPO PET imaging is lack of specificity of TSPO binding for activated microglia. TSPO is expressed on multiple different immune cells, including astrocytes, with TSPO radiotracer uptake reflecting activated astrocytes as well as activated microglia (21). Given the differing roles of microglia and astrocytes in neuroinflammatory responses, the result is a significant limitation on data interpretation and on defining the role of microglia in neurologic diseases. This lack of selectivity of TSPO radiotracers for microglia over other immune cells has led to the development of radiotracers that target alternative markers of microglial activation.

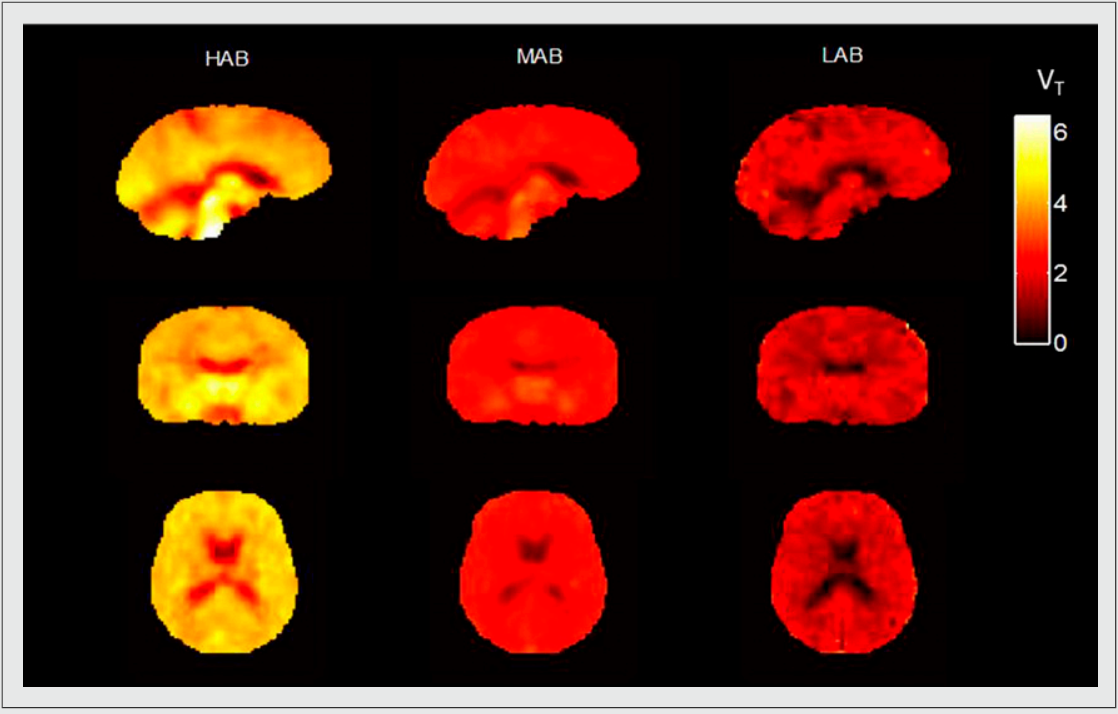

FIGURE 1. Parametric maps of ${ }^{18} \mathrm{~F}-\mathrm{PBR} 111$ volume of distribution in high-, mixed-, and low-affinity binders as determined by genotyping for rs6971 polymorphism. $\mathrm{HAB}=$ high-affinity binder; $\mathrm{LAB}=$ low-affinity binder; $\mathrm{MAB}=$ mixed-affinity binder; $\mathrm{V}_{\mathrm{T}}=$ volume of distribution. (Reprinted from (20).)
A further problem with the development of these newergeneration radiotracers is that those for which the best imaging results have been reported are ${ }^{11} \mathrm{C}$-labeled. However, a recent study found ${ }^{11} \mathrm{C}-\mathrm{PBR} 28$ and ${ }^{18} \mathrm{~F}$-PBR06 to be comparable with respect to binding affinity and background binding, suggesting that in future studies ${ }^{11} \mathrm{C}-\mathrm{PBR} 28$ can be substituted for ${ }^{18}$ F-PBR06 (22).

Lastly, a common problem with PET studies is the quantification of data. Qualitative assessment of images may be useful in cases of substantial or widespread neuroinflammation but may not show subtler changes in neuroinflammation. For this reason, pharmacokinetic modeling of receptor-radiotracer kinetics is often performed, although with practical limitations for application to routine clinical practice. Pharmacokinetic modeling requires either serial arterial blood samples or a suitable reference region where the radiotracer does not bind. Recently, however, the cerebellum was validated as a reference region for ${ }^{11} \mathrm{C}$-PBR28-TSPO binding in Alzheimer disease patients (8), potentially circumventing the need for arterial blood sampling for quantification of receptor binding in future studies of this disease using ${ }^{11} \mathrm{C}$-PBR28. However, because of differences in both radiotracer pharmacokinetics and TSPO distribution in diseases, it is necessary to independently validate a reference region for each radiotracer and in each disease being studied. Further, for diseases in which neuroinflammation is more diffuse and an unaffected anatomic reference region cannot be determined, alternative quantification methods are required, such as cluster analysis for the identification of an appropriate reference region.

\section{ALTERNATIVES TO TSPO IMAGING}

A limitation of TSPO as a biomarker of neuroinflammation is its expression on multiple types of immune cells. The development of more selective biomarkers of various participants in neuroinflammatory processes, including cell types such as monocytes, b cells, t cells, and astrocytes, as well molecular mediators of neuroinflammation, which can be targeted with PET radiotracers, would be of great value to the field. ${ }^{11} \mathrm{C}$-ketoprofen methyl ester specifically targets cyclooxygenase 1 and has been shown to be safe and to have favorable imaging characteristics warranting further investigation (23). Similarly, ${ }^{11} \mathrm{C}-\mathrm{RSR}-056$ and ${ }^{11} \mathrm{C}-\mathrm{A}-836339$ have shown promise as specific radiotracers for the cannabinoid type 2 receptor $(24,25)$.

The role of microglia in neuroinflammation is complex, with a growing body of evidence supporting two subtypes of microglia, one that is broadly neurotoxic and another that is broadly neuroprotective. Therefore, a PET radiotracer that is specific for either of these subtypes 
of microglia would be of great value in research and potentially in the clinic. However, there is currently no clear target that appears viable for a PET radiotracer that can distinguish between the microglia subtypes.

\section{CONCLUSION}

For in vivo TSPO imaging, an array of promising new radiotracers has been developed that overcome many of the limitations of ${ }^{11} \mathrm{C}-\mathrm{PK} 11195$. Recent recognition of the problem of there being various binding states for these tracers among the population has been mitigated by a simple blood test to ascertain binding status. However, there is as yet no ideal TSPO PET radiotracer for those who have genetically determined low binder status. Additional studies are required to further investigate the specificity of these radiotracers for activated microglia over other activated glial cells. More research is also required in preclinical and early clinical evaluation of these tracers, particularly those radiolabeled with ${ }^{18} \mathrm{~F}$, in order to establish their utility as research and clinical tools. However, early studies show that these tracers promise to be valuable tools for the investigation of a variety of neurologic diseases, including stroke, Alzheimer disease, and multiple sclerosis. More widespread use of these radiotracers will enable further novel insights into the role of neuroinflammation in the pathogenesis of many neurologic, psychiatric, and oncologic diseases. Of great potential is the future use of these radiotracers as diagnostic and prognostic tools and in assessing the effects of therapeutic interventions on disease activity.

\section{DISCLOSURE}

No potential conflict of interest relevant to this article was reported.

\section{REFERENCES}

1. Rupprecht R, Papadopoulos V, Rammes G, et al. Translocator protein (18 kDa) (TSPO) as a therapeutic target for neurological and psychiatric disorders. Nat Rev Drug Discov. 2010;9:971-988.

2. Scarf AM, Kassiou M. The translocator protein. J Nucl Med. 2011;52:677-680.

3. Owen DR, Matthews PM. Imaging brain microglial activation using positron emission tomography and translocator protein-specific radioligands. Int Rev Neurobiol. 2011;101:19-39.

4. Trapani A, Palazzo C, de Candia M, Lasorsa FM, Trapani G. Targeting of the translocator protein $18 \mathrm{kDa}$ (TSPO): a valuable approach for nuclear and optical imaging of activated microglia. Bioconjug Chem. 2013;24:1415-1428.

5. Buck JR, McKinley ET, Hight MR, et al. Quantitative, preclinical PET of translocator protein expression in glioma using ${ }^{18} \mathrm{~F}-\mathrm{N}$-fluoroacetyl- $\mathrm{N}$-(2,5dimethoxybenzyl)-2-phenoxyaniline. J Nucl Med. 2011;52:107-114.
6. Tang D, Hight MR, McKinley ET, et al. Quantitative preclinical imaging of TSPO expression in glioma using $\mathrm{N}, \mathrm{N}$-diethyl-2-(2-(4-(2-18 $\mathrm{F}$-fluoroethoxy)phenyl)-5,7dimethylpyrazolo[1,5-a]pyrimi din-3-yl)acetamide. J Nucl Med. 2012;53:287-294.

7. James ML, Belichenko NP, Nguyen TV, et al. PET imaging of translocator protein $(18 \mathrm{kDa})$ in a mouse model of Alzheimer's disease using $N$ - $(2,5$ dimethoxybenzyl)-2- ${ }^{18}$ F-fluoro- $\mathrm{N}$-(2-phenoxyphenyl)acetamide. $\mathrm{J} \mathrm{Nucl}$ Med. 2015;56:311-316.

8. Lyoo CH, Ikawa M, Liow JS, et al. Cerebellum can serve as a pseudo-reference region in Alzheimer disease to detect neuroinflammation measured with PET radioligand binding to translocator protein. J Nucl Med. 2015;56:701-706.

9. Gulyás B, Vas A, Toth M, et al. Age and disease related changes in the translocator protein (TSPO) system in the human brain: positron emission tomography measurements with $\left[{ }^{11} \mathrm{C}\right]$ vinpocetine. Neuroimage. 2011;56:1111-1121.

10. Varrone A, Mattsson P, Forsberg A, et al. In vivo imaging of the $18-\mathrm{kDa}$ translocator protein (TSPO) with $\left[{ }^{18}\right.$ F]FEDAA1 106 and PET does not show increased binding in Alzheimer's disease patients. Eur J Nucl Med Mol Imaging. 2013;40:921-931.

11. Yui J, Maeda J, Kumata K, et al. ${ }^{18}$ F-FEAC and ${ }^{18}$ F-FEDAC: PET of the monkey brain and imaging of translocator protein $(18 \mathrm{kDa})$ in the infarcted rat brain. J Nucl Med. 2010;51:1301-1309.

12. Gulyas B, Toth M, Vas A, et al. Visualising neuroinflammation in post-stroke patients: a comparative PET study with the TSPO molecular imaging biomarkers $\left[{ }^{11} \mathrm{C}\right] \mathrm{PK} 11195$ and $\left[{ }^{11} \mathrm{C}\right]$ vinpocetine. Curr Radiopharm. 2012;5:19-28.

13. Xie L, Yamasaki T, Ichimaru N, et al. $\left[{ }^{11} \mathrm{C}\right] \mathrm{DAC}-\mathrm{PET}$ for noninvasively monitoring neuroinflammation and immunosuppressive therapy efficacy in rat experimental autoimmune encephalomyelitis model. J Neuroimmune Pharmacol. 2012;7:231-242.

14. Airas L, Dickens AM, Elo P, et al. In vivo PET imaging demonstrates diminished microglial activation after fingolimod treatment in an animal model of multiple sclerosis. J Nucl Med. 2015;56:305-310.

15. Colasanti A, Guo Q, Muhlert N, et al. In vivo assessment of brain white matter inflammation in multiple sclerosis with ${ }^{18}$ F-PBR111 PET. $J$ Nucl Med. 2014;55: 1112-1118.

16. Oh U, Fujita M, Ikonomidou VN, et al. Translocator protein PET imaging for glial activation in multiple sclerosis. $J$ Neuroimmune Pharmacol. 2011;6:354-361.

17. Takano A, Piehl F, Hillert J, et al. In vivo TSPO imaging in patients with multiple sclerosis: a brain PET study with $\left[{ }^{18}\right.$ F]FEDAA1106. EJNMMI Res. 2013;3:30.

18. Owen DR, Gunn RN, Rabiner EA, et al. Mixed-affinity binding in humans with 18-kDa translocator protein ligands. J Nucl Med. 2011;52:24-32.

19. Owen DR, Yeo AJ, Gunn RN, et al. An 18-kDa translocator protein (TSPO) polymorphism explains differences in binding affinity of the PET radioligand PBR28. J Cereb Blood Flow Metab. 2012;32:1-5.

20. Guo Q, Colasanti A, Owen DR, et al. Quantification of the specific translocator protein signal of ${ }^{18} \mathrm{~F}-\mathrm{PBR} 111$ in healthy humans: a genetic polymorphism effect on in vivo binding. J Nucl Med. 2013;54:1915-1923.

21. Lavisse S, Guillermier M, Herard AS, et al. Reactive astrocytes overexpress TSPO and are detected by TSPO positron emission tomography imaging. J Neurosci. 2012;32:10809-10818.

22. Dickstein LP, Zoghbi SS, Fujimura Y, et al. Comparison of ${ }^{18} \mathrm{~F}$ - and ${ }^{11} \mathrm{C}$-labeled aryloxyanilide analogs to measure translocator protein in human brain using positron emission tomography. Eur J Nucl Med Mol Imaging. 2011;38:352-357.

23. Ohnishi A, Senda M, Yamane T, et al. Human whole-body biodistribution and dosimetry of a new PET tracer, $\left[{ }^{11} \mathrm{C}\right]$ ketoprofen methyl ester, for imagings of neuroinflammation. Nucl Med Biol. 2014;41:594-599.

24. Savonenko AV, Melnikova T, Wang Y, et al. Cannabinoid CB2 receptors in a mouse model of Abeta amyloidosis: immunohistochemical analysis and suitability as a PET biomarker of neuroinflammation. PLoS One. 2015;10:e129618.

25. Slavik R, Herde AM, Bieri D, et al. Synthesis, radiolabeling and evaluation of novel 4-oxo-quinoline derivatives as PET tracers for imaging cannabinoid type 2 receptor. Eur J Med Chem. 2015;92:554-564. 